\title{
Health Education program to improve the knowledge of Elderly Patients with Hip Fracture
}

\author{
Shimaa H Abd El-fatah, Elshazly S Mousa, Neama El-magrabi \& Nermeen M Abd El-Aziz. \\ Assistant lecturer, Gerontological Nursing, Faculty of Nursing, Sohag, University, Egypt. \\ Professor, Orthopedic Surgery, Faculty of Medicine, Sohag University, Egypt. \\ Assistant professor, Community Health Nursing, Faculty of Nursing-Assiut University, Egypt. \\ Lecturer of Gerontological Nursing, Faculty of Nursing, Assiut University, Egypt.
}

\begin{abstract}
Hip fracture is a serious injury in older people and can contribute to their death or loss of independence. Aim Evaluate the effect of health education program on Improve the knowledge of elderly patients with hip fracture. Research design: Quasi-experimental study was conducted in orthopedic surgery department at Sohag University Hospital. Purposive sample, their number was 76; they divided into two group study and control equal number 38. Data was collected from September 2014 to September 2015. Tools: two tools were used in the present study; a structured interview questionnaire, tool (I): to assess personal characteristics Tool (II): assess patient's knowledge regarding hip fracture. Results: About two thirds of patients aged between 60 - 70 years, more than half of them were females, most common type of hip fracture was intertrochanteric fracture, and main cause of hip fracture is fall down. Also there is a statistical significant difference between study and control groups regarding to knowledge after implement of health educational program. Conclusion: There is a statistical significant difference between study and control groups regarding knowledge after implement of health educational program. Recommendations Expanded health education about the problem for elders at Sohag through home visits.
\end{abstract}

Key Words: Hip Fracture, Elderly, Health Educational Program \& Knowledge

\section{Introduction}

An elderly person is an individual aged sixty-five years, considering that this age consistent with retirement age in most of the countries all over the world, but retirement age for most workers in Egypt is the age of sixty for the purposes of retirement and social insurance entitlement and pensions. The results of the general census of the population during the past two decades that the proportion of seniors $(60$ years and above) rose from $5.6 \%$ to $6.1 \%$ between the censuses 1986 and 2006 (Hassan, 2015).

In Egypt, the report of Egyptian Central Agency for Public Mobilization and Statistics (CAMPS), older adults aged 65 years and above represent $4.4 \%$ from total population. Also male life expectancy is 69.4 years and female life expectancy is 72.1 years. In Egypt, life expectancy at birth in Egypt gained 10 years from the period 1980-1985 to the period 20052010 , increasing from 59.9 years to 69.9 years. It is expected to reach 77.3 years in 2045-2050. The proportion of the elderly population $(65+)$ has also been increasing and is expected to reach $12.3 \%$ in 2050 (CAMPS, 2015).

With this increase in the aged population, there is an accompanying increase in prevalence of chronic conditions such as arthritis, cardiac disease, diabetes, hypertension, and obesity. They have multiple symptoms due to decline in the functioning of various body functions. This decrease in health status is mainly responsible for one of the most common and serious public health problems, namely falls. Over $33 \%$ of community-dwelling people aged over 65 years fall at least once a year, and of those $50 \%$ will have recurrent falls (Beaupre et al., 2013, Colquhoun et al., 2014 \& Hassan, 2015).

Hip fractures, also termed proximal femoral fractures, are a major cause of illness, disability and death in the elderly people, with an incidence increasing with age. Because the world's population is aging, the frequency of hip fractures is increasing by $1-3 \%$ per year in most areas of the world (Dailiana et al., 2013 \& Nagai \& Okawa, 2016).

Hip fractures can be subdivided into intracapsular (also termed subcapital and transcervical) fractures and extracapsular (also termed trochanteric, intertrochanteric and subtrochanteric) fractures. Intracapsular fractures are those occurring proximal to the attachment of the hip joint capsule to the femur at the intertrochanteric line. Extracapsular fractures are those which traverse the femur within the area of bone bounded by the intertrochanteric line proximally up to a distance of five centimeters from the distal part of the lesser trochanter (Handoll \& Parker, 2008 \& Yoon et al., 2011).

According to report of the American Academy of Orthopedic Surgeons (AAOS), Hip fractures most commonly occur from a fall or from a direct blow to 
the side of the hip. Some medical conditions such as osteoporosis, cancer, or stress injuries can weaken the bone and make the hip more susceptible to breaking. In severe cases, it is possible for the hip to break with the patient merely standing on the leg and twisting (AAOS, 2015).

Once the diagnosis of the hip fracture has been made, the patient's overall health and medical condition will be evaluated. Deciding between a surgical or conservative approach is the initial phase of any treatment plan. No operative management is generally reserved for patients with very high operative risk or limited life expectancies or those who are bedridden at baseline. The surgical intervention chosen depends on the type of fracture, the preference of the surgeon, the severity of the injury, the age of the patient, and the prognosis for recovery. The overall goal of surgery is to return patients to their prior level of functioning. In the short term, surgery also provides pain relief and allows for early mobilization (Anderson \& Wolfe, 2013).

The primary goal of nursing care for the older adult with hip fracture remains to maximize mobility and to preserve optimal function. The individual patient goal will partly be determined by pre-admission mobility and functional status. If the patient was bed or chair bound prior to surgery, pain and symptom management as well as preservation of that baseline may be the primary goal of postoperative care. For the individual who was mobile pre-fracture, postoperative mobility is critical to recovery (Maher et al., 2012).

\section{Significance of the study}

Hip fracture is a devastating injury for both patient and family - often resulting in impaired mobility, increased reliance on others, diminished health and sometimes death. Worldwide, the incidence of all hip fractures is 80 per 100000 individuals; Increase in the average lifespan has greatly increased the incidence of these fractures. Almost nine out of 10 proximal femur fractures occur in patients older than 65 years of age, and about three out of four occur in women. As many as $28 \%$ of older hip fracture patients die within one year of fracture and, of those that survive, it is estimated that between $24 \%$ to $75 \%$ will not return to their pre-fracture level of independence (El-Ghandour, 2014).

The increase in the older population is seen worldwide. Older people are at particular risk of hip fracture. Hip fracture is a significant problem and studies investigating health educational program for elderly with hip fracture and its effect on knowledge among Egyptian elderly people are uncommon. So, this study was conducted to determine the knowledge among a sample of elder Egyptian population and develop health educational program to improve the knowledge of elderly patients with hip fracture.

\section{Aim of the study}

Evaluate the effect of health education program on improve the knowledge of elderly patients with hip fracture.

\section{Research hypothesis}

Elderly patients with hip fracture attending educational program will exhibited a positive effect on their knowledge.

\section{Subjects \& Methods \\ Research design: \\ Quasi-experimental research design was utilized in this study \\ Setting}

The study was conducted in the inpatient orthopedic department and follow up in the orthopedic outpatient clinic at Sohag University Hospital.

\section{Subjects}

The study included a purposive sample of elderly patients attending to orthopedic department for one year who had hip fracture, their number were (76) elderly person aged 60 years and above. They were divided into two groups study and control group; group I used as study group and group II used as control group and equal number for each one (38).

\section{Inclusion criteria}

(A) 60 years and older

(B) Free from communication problems (speech and hearing problems)

(C) Accept to participate in the study.

\section{Tools for data collection}

Tool I: A structured interview questionnaire: It was developed by the researcher to collect the necessary data. It consisted of two parts:

Part (I): personal characteristics of the elderly patients: Entails data about demographic characteristic of the studied elderly such as; age, gender, residence, level of education, occupation, marital status, and living.

Part (II): Medical history; full history was taken from elderly about present history.

Present history: Side of fracture, type of fracture, causes of fracture, place of fall, cause of fall, time from injury to hospital arrival, time from injury to surgery (day), complications after surgery, and discharge.

Tool II: Assessment of patient's knowledge:

Assessment of patient's knowledge regarding hip fracture which include; definition, risk factors, causes, signs and symptoms, types, complication, medication, the ideal time for getting back to walking, the warning signs for calling the doctor, first aids, prevention of recurrent of fracture, components 
of balanced diet, nutrition for healing bone \& foods rich by calcium. Which includes 14 items, categorized, and scored Patients' knowledge; correct response was given 1 and was given incorrect zero. The score of each item summed-up and then converted into percent score (Poor $=$ score $<50 \%$, faired $=$ score $50-65 \%$, and good $=$ score $>65 \%$ ) (Abd-Elmohsen, 2013).

\section{Preparatory phase}

An official letter approval was obtained from the Dean of Faculty of Nursing at Sohag University to the director of Sohag University Hospital to obtain the necessary approval to conduct the study. This letter included a permission to collect the necessary data and explain the purpose and nature of the study.

\section{Content validity}

The tools were tested for content validity by five experts from the Faculty of Nursing (Community and Geriatric Nursing Departments) and Faculty of Medicine (Orthopedic Surgery Department). These experts assessed the tool for clarity, relevance, comprehensiveness, applicability, and understanding. All recommended modifications in the tools were done.

Pilot study

Before performing the main study, a pilot study was carried out on 5 elderly patients attended to orthopedic department to test the clarity of the questionnaire and to do the necessary modification. Also to estimate the time needed.

\section{Ethical considerations}

An informed consent for participation in the study was taken verbally from elderly persons after full explanation of the aim of the study. They were informed that their participation in this study was voluntarily. The elderly persons were given the opportunity to refuse participation, and they were notified that they could withdraw at any stage of the data collection without giving any reason. The subjects were also assured that any information collected would be confidential and used for the research purpose only. Also the study protocol and tools were approved by the Faculty Ethical Research Committee.

\section{Procedure/ Data Collection}

The researcher develops the program through four stages (assessment, planning, implementation and evaluation phase).

\section{A. Assessment phase}

At initial interview the researcher introduced herself to elderly to initiate line of communication, explain the nature and purpose of educational program, then filled out the questionnaire sheets before implementation the program to assess knowledge and needs of elderly.

\section{B. Planning phase General objective}

To provide elderly patients with right knowledge about hip fracture.

\section{Specific objectives}

After completing this program; the elderly with hip fracture will be able to

- Describe age related to changes on bone.

- Define of hip fracture.

- Enumerate signs and symptoms associated with hip fracture.

- Explain the risk factors and causes of hip fracture.

- List the various methods for diagnosis of hip fracture

- Demonstrate different types of exercises before surgery.

- Mention complications after hip fracture surgery $\&$ how to deal with it.

- Explain essential steps in home to avoid recurrent falling.

- Describe essential characteristics of balanced diet.

- Illustrate different types of exercises after surgery.

- Define the right way of prevention from recurrent fracture

The arrangement of conducting the program was done during this phase. The sessions and time of the program were decided. The chosen elderly patients were divided into study and control group. Other facilities were checked and arranged during this phase as teaching place, audiovisual aids and handout.

Teaching time: the time of teaching was decided according to coordination between the researcher and each elderly patient individually.

Teaching place: The study program was conducted in the inpatient orthopedic department at Sohag University Hospital.

Teaching methods and materials: it was important, before implementing the educational program, to prepare simple teaching instruments and audiovisual aids to be used; as lecture, discussion, brainstorming, picture and handout.

\section{Implementation phase}

The health educational program conducting during the period from starting September 2014 to end August 2015, three days weekly, the average number which interviewed was 1-2 elderly per week. The approximate time spent during filling of sheet was around 30-45 minutes according to respond of patient.

Implement the educational program for each elderly patient individually on study group, at orthopedic department and the total number of session was (7). Before beginning of the first session, an orientation to the program and its purpose was done and each 
patient elderly informed about time of session taken. second session about overview of hip fracture, third session about skills needed before operation, fourth session about complication \& warning signs after hip fracture surgery, fifth session about rehabilitation, sixth session about safety at home, first aids\& fall prevention, and seventh session summary for all units of the course. Each session started by summary about what was given during previous sessions and objectives of the new topics. Finally, the post test for patient's knowledge was implemented by using the same format of the pretest each session to determine the effect of the implemented program. Control group patients were exposed to the routine hospital nursing intervention.

\section{Evaluation phase}

After implementing the educational program for elderly patients, reassessment was done by the posttest to assess participant's knowledge.

\section{Statistical design}

The data obtained were reviewed, prepared for computer entry, coded, analyzed and tabulated. Descriptive statistics (i.e. frequencies, percentages, mean and standard deviation) were done using SPSS version 20. Chi-square and t-test used to compare differences in the distribution of frequencies among different groups. $\mathrm{P}$ value of less than 0.05 was considered significant.

\section{Results}

Table (1): Distribution of personal characteristics for elderly patients at Sohag University Hospital, 2014 2015.

\begin{tabular}{|c|c|c|c|c|c|c|}
\hline \multirow{3}{*}{ Personal characteristics } & \multicolumn{4}{|c|}{ Groups } & \multirow{3}{*}{$\begin{array}{c}\mathbf{X}^{2} \\
\text { Test }\end{array}$} & \multirow{3}{*}{ P-value } \\
\hline & \multicolumn{2}{|c|}{ Study (n=38) } & \multicolumn{2}{|c|}{ Control $(n=38)$} & & \\
\hline & No. & $\%$ & No. & $\%$ & & \\
\hline $\begin{array}{l}\text { 1. Age (years) } \\
60- \\
70- \\
\geq 80\end{array}$ & $\begin{array}{c}26 \\
10 \\
2 \\
\end{array}$ & $\begin{array}{c}68.4 \\
26.3 \\
5.3 \\
\end{array}$ & $\begin{array}{c}16 \\
14 \\
8\end{array}$ & $\begin{array}{l}42.1 \\
36.8 \\
21.1 \\
\end{array}$ & 3.324 & $\begin{array}{c}0.190 \\
\text { NS }\end{array}$ \\
\hline $\begin{array}{l}\text { 2. Gender } \\
\text { Male } \\
\text { Female }\end{array}$ & $\begin{array}{l}20 \\
18\end{array}$ & $\begin{array}{l}52.6 \\
47.4\end{array}$ & $\begin{array}{l}16 \\
22\end{array}$ & $\begin{array}{l}42.1 \\
57.9\end{array}$ & 0.422 & $\begin{array}{c}0.516 \\
\text { NS }\end{array}$ \\
\hline $\begin{array}{l}\text { 3. Educational level } \\
\text { Educated } \\
\text { Uneducated }\end{array}$ & $\begin{array}{c}2 \\
36\end{array}$ & $\begin{array}{c}5.3 \\
94.7\end{array}$ & $\begin{array}{c}4 \\
34\end{array}$ & $\begin{array}{l}10.5 \\
89.5\end{array}$ & 2.810 & $\begin{array}{c}0.590 \\
\text { NS }\end{array}$ \\
\hline $\begin{array}{l}\text { 4. Current Occupation } \\
\text { Worked without cash } \\
\text { Worked for cash }\end{array}$ & $\begin{array}{c}34 \\
4\end{array}$ & $\begin{array}{l}89.5 \\
10.5\end{array}$ & $\begin{array}{c}32 \\
6\end{array}$ & $\begin{array}{l}84.2 \\
15.8\end{array}$ & 3.226 & $\begin{array}{c}0.358 \\
\text { NS }\end{array}$ \\
\hline $\begin{array}{l}\text { 5. Marital status } \\
\text { Single } \\
\text { Married } \\
\text { Divorced } \\
\text { Widow } \\
\end{array}$ & $\begin{array}{c}2 \\
22 \\
0 \\
14\end{array}$ & $\begin{array}{c}5.3 \\
57.9 \\
0.0 \\
36.8 \\
\end{array}$ & $\begin{array}{c}2 \\
20 \\
2 \\
14\end{array}$ & $\begin{array}{c}5.3 \\
52.6 \\
5.3 \\
36.8 \\
\end{array}$ & 1.048 & $\begin{array}{c}0.790 \\
\text { NS }\end{array}$ \\
\hline $\begin{array}{l}\text { 6. Residence } \\
\text { Urban } \\
\text { Rural } \\
\end{array}$ & $\begin{array}{l}12 \\
26 \\
\end{array}$ & $\begin{array}{l}31.6 \\
68.4 \\
\end{array}$ & $\begin{array}{l}14 \\
24\end{array}$ & $\begin{array}{l}36.8 \\
63.2 \\
\end{array}$ & 0.117 & $\begin{array}{c}0.732 \\
\text { NS }\end{array}$ \\
\hline $\begin{array}{l}\text { 7. Living status } \\
\text { with Family } \\
\text { alone }\end{array}$ & $\begin{array}{c}38 \\
0\end{array}$ & $\begin{array}{c}100.0 \\
0.0\end{array}$ & $\begin{array}{c}34 \\
4\end{array}$ & $\begin{array}{l}89.5 \\
10.5\end{array}$ & 2.140 & $\begin{array}{c}0.146 \\
\text { NS }\end{array}$ \\
\hline
\end{tabular}


Table (2): Distribution of present history for elderly patients at Sohag University Hospital, 2014 - 2015.

\begin{tabular}{|c|c|c|c|c|c|c|}
\hline \multirow{3}{*}{ Present history } & \multicolumn{4}{|c|}{ Groups } & \multirow{3}{*}{$\begin{array}{c}\mathbf{X}^{2} \\
\text { Test }\end{array}$} & \multirow{3}{*}{ P-value } \\
\hline & \multicolumn{2}{|c|}{$\begin{array}{c}\text { Study } \\
(n=38)\end{array}$} & \multicolumn{2}{|c|}{$\begin{array}{c}\text { Control } \\
(n=38)\end{array}$} & & \\
\hline & No. & $\%$ & No. & $\%$ & & \\
\hline $\begin{array}{l}\text { 1. Side of hip fracture } \\
\text { - Right } \\
\text { - Left }\end{array}$ & $\begin{array}{l}22 \\
16\end{array}$ & $\begin{array}{l}57.9 \\
42.1\end{array}$ & $\begin{array}{l}22 \\
16\end{array}$ & $\begin{array}{l}57.9 \\
42.1\end{array}$ & 0.000 & $\begin{array}{l}1.0 \\
\mathrm{NS}\end{array}$ \\
\hline $\begin{array}{l}\text { 2. Type of hip fractures } \\
\text { - Femoral neck. } \\
\text { - Intertrochanteric } \\
\text { - Subtrochanteric }\end{array}$ & $\begin{array}{c}12 \\
22 \\
4\end{array}$ & $\begin{array}{l}31.6 \\
57.9 \\
10.5\end{array}$ & $\begin{array}{c}16 \\
18 \\
4\end{array}$ & $\begin{array}{l}42.1 \\
47.4 \\
10.5\end{array}$ & 0.486 & $\begin{array}{c}0.784 \\
\mathrm{NS}\end{array}$ \\
\hline $\begin{array}{l}\text { 3. Causes of hip fracture } \\
\text { - Accident } \\
\text { - } \quad \text { Fall }\end{array}$ & $\begin{array}{l}10 \\
28 \\
\end{array}$ & $\begin{array}{l}26.3 \\
73.7 \\
\end{array}$ & $\begin{array}{c}8 \\
30 \\
\end{array}$ & $\begin{array}{l}21.0 \\
79.0 \\
\end{array}$ & 0.146 & $\begin{array}{c}0.703 \\
\text { NS }\end{array}$ \\
\hline $\begin{array}{ll}\text { A. } & \text { Place of fall } \\
\text { - } & \text { Home } \\
\text { - } & \text { Work-place/ office } \\
\text { - } & \text { Passage }\end{array}$ & $\begin{array}{c}26 \\
2 \\
0\end{array}$ & $\begin{array}{c}92.9 \\
7.1 \\
0.00\end{array}$ & $\begin{array}{c}22 \\
2 \\
6\end{array}$ & $\begin{array}{c}73.3 \\
6.7 \\
20.00\end{array}$ & 3.136 & $\begin{array}{l}0.208 \\
\text { NS }\end{array}$ \\
\hline $\begin{aligned} & \text { B. Causes of fall } \\
& \text { - } \text { Slipped } \\
& \text { - } \text { Lost balance } \\
& \text { - } \text { felt faint/dizzy } \\
& \text { - } \text { Unknown }\end{aligned}$ & $\begin{array}{l}16 \\
4 \\
8 \\
0\end{array}$ & $\begin{array}{l}57.1 \\
14.3 \\
28.6 \\
0.00\end{array}$ & $\begin{array}{c}12 \\
10 \\
6 \\
2\end{array}$ & $\begin{array}{l}40.0 \\
33.3 \\
20.0 \\
6.7\end{array}$ & 2.683 & $\begin{array}{c}0.443 \\
\mathrm{NS}\end{array}$ \\
\hline $\begin{array}{l}\text { 4. Time from fracture } \\
\text { to hospital arrival } \\
\text { - } \quad<24 \mathrm{hrs} . \\
\text { - } \quad>24 \mathrm{hrs}\end{array}$ & $\begin{array}{l}28 \\
10\end{array}$ & $\begin{array}{l}73.7 \\
26.3\end{array}$ & $\begin{array}{l}28 \\
10\end{array}$ & $\begin{array}{l}73.7 \\
26.3\end{array}$ & 0.000 & $\begin{array}{l}1.0 \\
\mathrm{NS}\end{array}$ \\
\hline $\begin{array}{l}\text { 5. Time from fracture to } \\
\text { operation (day) } \\
\text { - Within day } \\
\text { - } 1-2 \\
\text { - } \quad>2\end{array}$ & $\begin{array}{c}0 \\
10 \\
28\end{array}$ & $\begin{array}{l}0.00 \\
26.3 \\
73.7\end{array}$ & $\begin{array}{c}8 \\
6 \\
24\end{array}$ & $\begin{array}{l}21.0 \\
15.8 \\
63.2\end{array}$ & 5.416 & $\begin{array}{c}0.067 \\
\text { NS }\end{array}$ \\
\hline $\begin{array}{l}\text { 6. Discharge to ( place) } \\
\text { - } \quad \text { Home } \\
\text { - } \quad \text { Other acute hospital ward } \\
\text { - } \quad \text { In-hospital death }\end{array}$ & $\begin{array}{c}32 \\
4 \\
2\end{array}$ & $\begin{array}{c}84.2 \\
10.5 \\
5.3\end{array}$ & $\begin{array}{c}30 \\
6 \\
2\end{array}$ & $\begin{array}{c}79.0 \\
15.7 \\
5.3\end{array}$ & 0.232 & $\begin{array}{l}0.890 \\
\mathrm{NS}\end{array}$ \\
\hline $\begin{array}{l}\text { a. The causes of intra-hospital } \\
\text { death } \\
\text { - Circulatory disease } \\
\text { - } \quad \text { Respiratory disease }\end{array}$ & $\begin{array}{l}2 \\
0\end{array}$ & $\begin{array}{c}100.0 \\
0.0\end{array}$ & $\begin{array}{l}0 \\
2\end{array}$ & $\begin{array}{c}0.0 \\
100.0\end{array}$ & 2.00 & $\begin{array}{c}0.157 \\
\text { NS }\end{array}$ \\
\hline $\begin{array}{l}\text { 7. Walking ability } \\
\text { - without aid } \\
\text { - } \quad \text { with a cane }\end{array}$ & $\begin{array}{c}34 \\
4\end{array}$ & $\begin{array}{l}89.5 \\
10.5\end{array}$ & $\begin{array}{c}30 \\
8\end{array}$ & $\begin{array}{l}79.0 \\
21.0\end{array}$ & 0.792 & $\begin{array}{c}0.374 \\
\text { NS }\end{array}$ \\
\hline
\end{tabular}

Percentage calculated from those mentioned cause of fracture is falling or death at hospital. 
Table (3): Distribution of knowledge before program regarding their definition, risk factors, cause, signs \& symptoms and types of hip fracture for elderly patients at Sohag University Hospital, 2014 - 2015.

\begin{tabular}{|c|c|c|c|c|c|c|}
\hline \multirow{3}{*}{ Knowledge } & \multicolumn{4}{|c|}{ Groups } & \multirow{3}{*}{$\begin{array}{c}\mathbf{X}^{2} \\
\text { Test }\end{array}$} & \multirow{3}{*}{ P-value } \\
\hline & \multicolumn{2}{|c|}{$\begin{array}{l}\text { Study } \\
(n=38)\end{array}$} & \multicolumn{2}{|c|}{$\begin{array}{c}\text { Control } \\
(n=38)\end{array}$} & & \\
\hline & No. & $\%$ & No. & $\%$ & & \\
\hline $\begin{array}{l}\text { Definition of hip fracture } \\
\text { - Incorrect } \\
\text { - } \quad \text { Correct } \\
\end{array}$ & $\begin{array}{l}28 \\
10 \\
\end{array}$ & $\begin{array}{l}73.7 \\
26.3 \\
\end{array}$ & $\begin{array}{c}30 \\
8 \\
\end{array}$ & $\begin{array}{l}79.0 \\
21.0\end{array}$ & 0.146 & $0.703 \mathrm{SN}$ \\
\hline $\begin{array}{l}\text { Risk factors of hip fracture } \\
\text { - Incorrect } \\
\text { - } \quad \text { Correct }\end{array}$ & $\begin{array}{l}16 \\
22\end{array}$ & $\begin{array}{l}42.1 \\
57.9\end{array}$ & $\begin{array}{l}20 \\
18 \\
\end{array}$ & $\begin{array}{l}52.6 \\
47.4\end{array}$ & 1.057 & $0.589 \mathrm{NS}$ \\
\hline $\begin{array}{l}\text { Causes of hip fracture } \\
\text { - Incorrect } \\
\text { - } \quad \text { Correct } \\
\end{array}$ & $\begin{array}{l}14 \\
24 \\
\end{array}$ & $\begin{array}{l}36.8 \\
63.2 \\
\end{array}$ & $\begin{array}{l}12 \\
26 \\
\end{array}$ & $\begin{array}{l}31.6 \\
68.4 \\
\end{array}$ & 3.259 & $0.196 \mathrm{NS}$ \\
\hline $\begin{array}{l}\text { Signs of hip fracture } \\
\text { - Incorrect } \\
\text { - } \quad \text { Correct } \\
\end{array}$ & $\begin{array}{c}6 \\
32 \\
\end{array}$ & $\begin{array}{l}15.8 \\
84.2 \\
\end{array}$ & $\begin{array}{l}10 \\
28 \\
\end{array}$ & $\begin{array}{l}26.3 \\
73.7 \\
\end{array}$ & 4.389 & $0.111 \mathrm{NS}$ \\
\hline $\begin{array}{l}\text { Types of hip fracture } \\
\text { - Incorrect } \\
\text { - } \quad \text { Correct }\end{array}$ & $\begin{array}{l}24 \\
14\end{array}$ & $\begin{array}{l}63.2 \\
36.8\end{array}$ & $\begin{array}{c}30 \\
8\end{array}$ & $\begin{array}{l}79.0 \\
21.0\end{array}$ & 2.238 & $\begin{array}{c}0.327 \\
\text { NS }\end{array}$ \\
\hline
\end{tabular}

Table (4): Distribution of knowledge before program regarding to complications, ability of walking, cause of use of aspirin, warning signs and first aids for hip fracture for elderly patients at Sohag University Hospital, $2014-2015(N=76)$.

\begin{tabular}{|c|c|c|c|c|c|c|}
\hline \multirow{3}{*}{ Knowledge } & \multicolumn{4}{|c|}{ Groups } & \multirow{3}{*}{$\begin{array}{c}\mathbf{X}^{2} \\
\text { Test }\end{array}$} & \multirow{3}{*}{ P-value } \\
\hline & \multicolumn{2}{|c|}{$\begin{array}{c}\text { Study } \\
(\mathrm{n}=38)\end{array}$} & \multicolumn{2}{|c|}{$\begin{array}{c}\text { Control } \\
(\mathrm{n}=38)\end{array}$} & & \\
\hline & No. & $\%$ & No. & $\%$ & & \\
\hline $\begin{array}{l}\text { Complications of hip fracture } \\
\text { - } \quad \text { Incorrect } \\
\text { - } \quad \text { Correct } \\
\end{array}$ & $\begin{array}{c}32 \\
6 \\
\end{array}$ & $\begin{array}{l}84.2 \\
15.8 \\
\end{array}$ & $\begin{array}{c}32 \\
6 \\
\end{array}$ & $\begin{array}{l}84.2 \\
15.8 \\
\end{array}$ & 1.200 & $\begin{array}{c}0.549 \\
\text { NS }\end{array}$ \\
\hline $\begin{array}{l}\text { Ability to walk after surgery } \\
\text { - Incorrect } \\
\text { - } \quad \text { Correct }\end{array}$ & $\begin{array}{c}34 \\
4\end{array}$ & $\begin{array}{l}89.5 \\
10.5\end{array}$ & $\begin{array}{c}34 \\
4\end{array}$ & $\begin{array}{l}89.5 \\
10.5\end{array}$ & 0.000 & $1.00 \mathrm{NS}$ \\
\hline $\begin{array}{l}\text { Causes of use of aspirin after surgery } \\
\text { - Incorrect } \\
\text { - } \quad \text { Correct }\end{array}$ & $\begin{array}{l}24 \\
14\end{array}$ & $\begin{array}{l}63.2 \\
36.8\end{array}$ & $\begin{array}{l}22 \\
16\end{array}$ & $\begin{array}{l}57.9 \\
42.1\end{array}$ & 1.043 & $\begin{array}{c}0.593 \\
\text { NS }\end{array}$ \\
\hline $\begin{array}{l}\text { Warning Signs/to call physician } \\
\text { - Incorrect } \\
\text { - } \quad \text { Correct }\end{array}$ & $\begin{array}{c}6 \\
32 \\
\end{array}$ & $\begin{array}{l}15.8 \\
84.2\end{array}$ & $\begin{array}{c}8 \\
30\end{array}$ & $\begin{array}{l}21.0 \\
79.0\end{array}$ & 1.161 & $\begin{array}{c}0.560 \\
\text { NS }\end{array}$ \\
\hline $\begin{array}{l}\text { First aids for fracture } \\
\text { - } \quad \text { Incorrect } \\
\text { - } \quad \text { Correct }\end{array}$ & $\begin{array}{c}30 \\
8\end{array}$ & $\begin{array}{l}79.0 \\
21.0\end{array}$ & $\begin{array}{c}34 \\
4\end{array}$ & $\begin{array}{l}89.5 \\
10.5\end{array}$ & 0.792 & $\begin{array}{c}0.374 \\
\text { NS }\end{array}$ \\
\hline
\end{tabular}


Table (5): Distribution of knowledge before program regarding to prevention, element of balance diet, foods improve healing of bone and food rich by calcium for elderly patients at Sohag University Hospital, 2014 2015.

\begin{tabular}{|c|c|c|c|c|c|c|}
\hline \multirow{3}{*}{ Knowledge } & \multicolumn{4}{|c|}{ Groups } & \multirow{3}{*}{$\begin{array}{c}X^{2} \\
\text { Test }\end{array}$} & \multirow{3}{*}{ P-value } \\
\hline & \multicolumn{2}{|c|}{$\begin{array}{l}\begin{array}{l}\text { Study } \\
(\mathbf{n}=38)\end{array} \\
\end{array}$} & \multicolumn{2}{|c|}{$\begin{array}{c}\text { Control } \\
(\mathbf{n}=38)\end{array}$} & & \\
\hline & No. & $\%$ & No. & $\%$ & & \\
\hline $\begin{array}{l}\text { Prevention of hip fracture } \\
\text { - Incorrect } \\
\text { - Correct }\end{array}$ & $\begin{array}{l}28 \\
10\end{array}$ & $\begin{array}{l}73.7 \\
26.3\end{array}$ & $\begin{array}{c}30 \\
8\end{array}$ & $\begin{array}{l}79.0 \\
21.0\end{array}$ & 0.146 & $0.703 \mathrm{NS}$ \\
\hline $\begin{array}{l}\text { Element of balance diet } \\
\text { - Incorrect } \\
\text { - Correct }\end{array}$ & $\begin{array}{c}30 \\
8\end{array}$ & $\begin{array}{l}79.0 \\
21.0\end{array}$ & $\begin{array}{l}28 \\
10\end{array}$ & $\begin{array}{l}73.7 \\
26.3\end{array}$ & 5.467 & $0.065 \mathrm{NS}$ \\
\hline $\begin{array}{l}\text { Foods improve healing of bone } \\
\text { - Incorrect } \\
\text { - } \quad \text { Correct }\end{array}$ & $\begin{array}{c}34 \\
4\end{array}$ & $\begin{array}{l}89.5 \\
10.5\end{array}$ & $\begin{array}{c}30 \\
8\end{array}$ & $\begin{array}{l}79.0 \\
21.0\end{array}$ & 3.726 & $0.155 \mathrm{NS}$ \\
\hline $\begin{array}{l}\text { Food rich by calcium } \\
\text { - Incorrect } \\
\text { - Correct }\end{array}$ & $\begin{array}{l}28 \\
10\end{array}$ & $\begin{array}{l}73.7 \\
26.3\end{array}$ & $\begin{array}{l}26 \\
12\end{array}$ & $\begin{array}{l}68.4 \\
31.6\end{array}$ & 5.526 & $0.063 \mathrm{NS}$ \\
\hline
\end{tabular}

Table (6): Mean scores of knowledge for elderly patients before and after implementation of educational program at Sohag University Hospital, 2014 - 2015.

\begin{tabular}{|l|c|c|}
\hline \multicolumn{1}{|c|}{ Variable } & Pre-program & Post- program \\
\cline { 2 - 3 } & Mean \pm SD & Mean \pm SD \\
\hline Study group & $5.8 \pm 2.5$ & $8.7 \pm 4.5$ \\
\hline Control group & $5.4 \pm 1.9$ & $5.4 \pm 2.9$ \\
\hline P-value & 0.884 & $0.001^{*}$ \\
\hline
\end{tabular}

* Statistically significant $P<0.05$

Table (7): Comparison between both groups regarding knowledge score before and after implementation of educational program at Sohag University Hospital, 2014 - 2015.

\begin{tabular}{|c|c|c|c|c|c|c|c|c|c|c|c|c|}
\hline \multirow{4}{*}{$\begin{array}{c}\text { Knowledge } \\
\text { level }\end{array}$} & \multicolumn{8}{|c|}{ Groups } & \multirow{2}{*}{\multicolumn{2}{|c|}{$\begin{array}{c}\mathbf{X}^{2} \\
\text { Test }\end{array}$}} & \multirow{2}{*}{\multicolumn{2}{|c|}{ P-value }} \\
\hline & \multicolumn{4}{|c|}{$\begin{array}{l}\text { Study } \\
(n=38)\end{array}$} & \multicolumn{4}{|c|}{$\begin{array}{c}\text { Control } \\
(n=38)\end{array}$} & & & & \\
\hline & \multicolumn{2}{|c|}{ Pre } & \multicolumn{2}{|c|}{ Post } & \multicolumn{2}{|c|}{ Pre } & \multicolumn{2}{|c|}{ post } & \multirow{2}{*}{ pre } & \multirow{2}{*}{ post } & \multirow{2}{*}{ Pre } & \multirow{2}{*}{ Post } \\
\hline & No & $\%$ & No & $\%$ & No & $\%$ & No & $\%$ & & & & \\
\hline Poor & 32 & 84.2 & 4 & 10.5 & 32 & 84.2 & 32 & 84.2 & \multirow{4}{*}{0.00} & \multirow{4}{*}{48.96} & \multirow{4}{*}{1.000} & \multirow{4}{*}{$0.000 * *$} \\
\hline Faired & 6 & 15.8 & 2 & 5.3 & 6 & 15.8 & 6 & 15.8 & & & & \\
\hline Good & 0 & 0.00 & 32 & 84.2 & 0 & 0.00 & 0 & 0.00 & & & & \\
\hline Total & 38 & 100.0 & 38 & 100.0 & 38 & 100.0 & 38 & 100.0 & & & & \\
\hline
\end{tabular}

* Statistically significant $P<0.05$

Table (1): Clarifies that age, it was noticed that more than two thirds $(68.4 \%)$ of study group their age between 60 to less than 70 years compared to more than two fifths $(42.1 \%)$ of control group. regarding to sex, it was found that little more than half $(52.6 \%)$ of study group were males compared to more than two fifths $(42.1 \%)$ of control group while rest of them were females.
As regards educational level, it was observed that high percentage of elderly patients were uneducated (94.7\%) compared to $(89.5 \%)$ of control group. Also the finding shows that the majority of elderly patients were working without cash $89.5 \%$ compared to $84.2 \%$ of control group. Concerning on marital status, the present study showed that more than half $(57.9 \%)$ of study group were married compared to $52.6 \%$ of 
control group, while $5.3 \%$ only of them were single. Regarding to their residence, it was found that the about two thirds of them lived in rural areas. Also the finding shows that the majority of studies sample living with family.

Table (2): Presents that, more than half (57.9\%) of studied sample reported that right hip was affected, and rest of them left side affected. As regards type of fracture, it observed that $57.9 \%$ had Intertrochanteric fracture compared to $47.4 \%$ of control group, while $10.5 \%$ of them had subtrochanteric. As regard to cause of fracture; about three quarters of studied sample reported that cause of fracture is fall. In this study, place of fall, it found that the majority $(92.9 \%)$ of study group falling occurred at home compared to less than three quarters of control group. For cause of fall, it was noticed that main cause of falling is slipped, while $6.7 \%$ of control group only reported that didn't know cause of fall.

This table presents that, more than half $(57.9 \%)$ of studied sample reported that right hip was affected, and rest of them left side affected. As regards type of fracture, it observed that $57.9 \%$ had Intertrochanteric fracture compared to $47.4 \%$ of control group, while $10.5 \%$ of them had subtrochanteric. As regard to cause of fracture; about three quarters of studied sample reported that cause of fracture is fall. In this study, place of fall, it found that the majority (92.9\%) of study group falling occurred at home compared to less than three quarters of control group. For cause of fall, it was noticed that main cause of falling is slipped, while $6.7 \%$ of control group only reported that didn't know cause of fall.

In this study less than three quarters $(73.7 \%)$ of studied sample reported that they arrived at hospital within 24 hours from fracture, while rest of them arrived after 24 hours. Regarding time from hip fracture to operation, it was observed that less than three quarters of study group reported that the operation is performed after 2 days from hip fracture compared to $63.2 \%$ of control group. Concerning discharge it found that high percentage of studied sample discharged to home, while $5.3 \%$ only of them died at hospital, cause of death in the study group was circulatory diseases while in control group was respiratory disease. Regarding walking ability, the majority of studied sample $(89.5 \% \& 79.0 \%)$ reported that before fracture able to walk without aids.

Table (3): Illustrates that more one quarter (26.3\%) of the study group mention the meaning of hip fracture correctly compared to more one fifth (21.0\%) of control group. Regarding the risk factors of hip fracture, more than half $(57.9 \%)$ of study group had correct knowledge compared to less than $(47.4 \%)$ of control group.
Regarding causes of hip fracture less than two thirds $(63.2 \%)$ of study group had correct knowledge about causes of hip fracture compared to more than two thirds $(68.4 \%)$ of control group. Concerning signs \& symptoms of hip fracture, it was noticed that the high percentage of studied sample had correct knowledge, while nearly one quarters $(26.3 \%)$ of control group had incorrect knowledge about signs and symptoms of hip fracture. As regard to types of hip fracture, it was found that less than two thirds $(63.2 \%)$ of the study group had incorrect knowledge compared to more than three quarters of control group.

Table (4): Presents that the high percentages of studied sample mention incorrect knowledge about complications after hip fracture operation, while $15.8 \%$ only of them had correct knowledge. As regard ability to walk after surgery, the majority $(89.5 \%)$ of the studied sample had incorrect knowledge.

As regard cause of using of aspirin after surgery, it was noticed that less than two thirds $(63.2 \%)$ of the study group had incorrect knowledge compared to more than half $(57.9 \%)$ of control group. Warning signs to call physician, the high percentage $(84.2 \%)$ of study group had correct knowledge compared to $79.0 \%$ of control group. Regarding first aids for fracture, it observed that more than three quarters $(79.0 \%)$ of study group had incorrect knowledge compared to the majority $(89.5 \%)$ of control group.

Table (5): Reveals that less than three quarters (73.7\%) of study group doesn't know any method of prevention of hip fracture compared to more than three quarters $(79.9 \%)$. Regarding to element of balance diet, more than three quarters $(79.0 \%)$ of study group had incorrect answer, while $21.0 \%$ of control group identifies element of balance diet.

Concerning on foods improve healing of bone, it was observed that high percentage of studied sample had incorrect knowledge, while more than one fifth of control group had knowledge about Foods improve healing of bone. Regarding food rich by calcium about three quarters $(73.7 \%)$ of study group doesn't know compared to more than two thirds $(68.4 \%)$ of control group.

Table (6): Shows that, there were statistical significant differences before and after implementation of the educational program among study groups while non-statistical significant differences were noticed throughout the educational program among control group regarding level of knowledge about hip fracture operation.

Table (7): Presents the total scores of knowledge among elderly patients with hip fracture after implementing education program for both study and control groups. This table shows improved level of knowledge in study group that $(84.2 \%)$ of the studied 
group have poor level of knowledge before applied the program and become highest percentage after the program, while no improvement in control group. And demonstrates that, high statistical significant difference was found between study and control groups as regard patient's level of knowledge about hip fracture.

\section{Discussion}

As regards personal characteristics of the studied elderly patients, the finding of the present study showed that highest percentage of studied sample had age ranged between 60 - 70 years, while $5.3 \%$ of them aged 80 years or more. This finding similar to the result of Deka et al., (2015) who found that about two thirds $(62.5 \%)$ of studied sample had age ranged between 50 - 70 years. Also this result supported by Kiel et al., (2007) \& Vochteloo et al., (2013) who found that about $7.0 \%$ of the studied sample was 80 years and more. But, this finding disagrees with Paula et al., (2015) \& Griffin et al., (2016) who found that hip fracture was more prevalent among populations aged 80 years and more. This can be interpreted as the difference between the studies might be due to elderly patients with hip fracture with 80 years had co-morbidities diseases make them medical unstable to perform surgery so that excluded these patients from the present study because from inclusion criteria patient should perform surgery.

As regarding sex, less than half of study group $(47.4 \%)$ with hip fracture was females and the rest of them were males compared to $57.9 \%$ of control group. That means the incidence of hip fracture slightly higher in females than males. This result agrees with McGilton et al., (2015) who reported that $58.1 \%$ of the studied samples were females. Also this result supported by Zapatero et al., (2013), Liao et al., (2014) \& Zheng et al., (2016) who found in these studies the incidence of hip fracture slightly higher in females than males. The present study disagree with Keswani et al., (2016) who studied reported that $22 \%$ for men and $78 \%$ for women have hip fracture

Concerning educational level, it was observed that high percentage of the studied sample were uneducated. This finding is nearly similar to the results of Vergara et al., (2014) \& Liu et al., (2015) who showed that uneducated level the highest percentage in the studied sample. On the other hand, The present study disagree with Orwig et al., (2011) who studied "the delivery and outcomes of a yearlong home exercise program after hip fracture", and reported that more than half of the studied sample (56\%) were educated. This can be interpreted because about half of studied sample were females and in Egypt females less access to education.
Concerning marital status, the finding of present study showed that more than half of studied sample were married. This result agrees with Shyu et al., (2012) \& Liu et al., (2015), in Taiwan who found that more than half of studied sample were married. But, this finding disagree with the result of Orwig et al., (2011) who reported that less than one third $(31.0 \%)$ of the studied sample were married.

Concerning the place of residence, result reveals that about two thirds of elderly patients were living in rural areas and the rest of them were living in urban areas. The present study disagree with Herr \& Titler, (2009) \& Dailiana et al., (2013), who stated that about three quarters of the studied sample lived in urban areas.

Regarding living status, it was observed that the majority of the studied samples were living with family. The current result agrees with Kim et al., (2012), who reported that $86.3 \%$ of studied sample living with family. Also, this result supported with Maggi et al., (2010), who found that the majority of the studied sample living with family. On the other hand, the present study disagrees with Kondo et al., (2012) who stated that the majority $(85.8 \%)$ of the studied sample living with alone.

Regarding affected side of fracture; more than half $(57.6 \%)$ of studied sample their affected hip were right. This finding is consistent with the study conducted in the University of Bergen by Matre, (2013) who found that $54.5 \%$ of the studied sample the right hip is affected. This result also supported by McGilton et al., (2015) who reported that $57.1 \%$ of the studied sample right hip was affected.

Concerning type of fracture; the finding of present study revealed that more than half of the studied sample had intertrochanteric fracture, while $10.5 \%$ only of them had subtrochanteric fracture. This result agree with Makridis et al., (2014) \& Keswani et al., (2016), who reported that more than half of the studied sample had intertrochanteric fracture. On the other hand, this present study disagree with Shyu et al., (2012), who studied "Comprehensive care improves health outcomes among elderly Taiwanese patients with hip fracture" and found that (35.4\%) of studied sample had intertrochanteric fracture. the present result is supported with Maggi et al., (2010), Paula et al., (2015) \& Larsson et al., (2016) who reported that less than $10.0 \%$ of studied sample had subtrochanteric fracture. This finding indicates subtrochanteric fractures are rarely seen in the elderly because they are more likely to occur after highenergy trauma (accident).

As regards cause of fracture, it was found that the main cause of hip fracture among studied sample is falling. This finding resembles to the result of the study done in Jordanian population by Shakhatreh, 
(2001) who found that $95 \%$ of studied sample reported cause of the fracture is falling down. This result also supported by Worldwide studies which performed by Soveid et al., (2005), Hasegawa, (2007), Shyu et al., (2008) \& El-jedi et al., (2015) who found in these studies the main cause of hip fracture among elderly patients is falling down. This result due to elderly people had fragile bone and any simple fall lead to fracture.

Concerning place of fall, it was found that about three quarters $(73.3 \%)$ of the studied sample reported falling occurred at home. This result is nearly similar to the results of Al-Faisal, (2006) who reported that $(\mathbf{6 3 . 5 \%})$ of the studied sample had fracture occurred at home. As regard to cause of fall, it observed that main cause of the fall among the studied sample is slipped and faint. But, this result disagrees with Matsui et al., (2014) who found that main cause of the fall at studied sample is loss of balance and tripping.

As regards time from fracture to surgery, it was found that only $(21.0 \%)$ of studied sample were operated within day of fracture, while about three quarters of studied sample were operated after two days from admission. This finding is consistent with the study conducted at Gaza by El-jedi et al., (2015) who found that $27.5 \%$ of elderly patients were operated at the same day of fracture and rest of them $(72.5 \%)$ were operated after three days from admission.

On the other hand, the present study disagrees with a study conducted by Casaletto \& Gatt, (2004) \& (CIHI), (2015) who showed that $13.0 \%$ only of the studied sample waited two days and more. Also this finding contrast with Larsson et al., (2016) who stated that $(79 \%)$ of the studied sample performed the operation within the day. The waiting time before surgical intervention was long in the present study compared with other global studies; early surgery is not always feasible due to medical instability in older patients and the need to undertake a many clinical and laboratory investigation before hip fracture operation especially elderly patients.

Concerning discharge, the finding showed that the majority of the studied sample was discharged to home, while $5.3 \%$ only of them died during their hospital stay. This present study in same line with Tarazona - Santabalbina et al., (2012) who conducted that "Early interdisciplinary hospital intervention for elderly patients with hip fractures functional outcome and mortality" and reported that $63.7 \%$ of the studied samples return to home and $4.7 \%$ only of them died during their hospital stay. This finding consistent with other studies of Perneczky et al., (2006) \& McGilton et al., (2013) and CIHI, (2015) who reported that in these studies high percentage of the studied sample return to home.
Also this finding is consistent with the studies conducted in Netherlands \& Sweden by Vochteloo et al., (2013) \&Larsson et al., (2016) who found that $3.3 \% \& 5.0 \%$ of the studied sample respectively died in hospital. On other hand the present study disagrees with Matre, (2013) who reported that $11.9 \%$ only of the studied sample discharged to home. This can be interpreted as the difference between the studies might be due to the cultural expectations of filial piety that is commonly encountered in Africa countries, and the ease of employing domestic helpers who are able to help with the provision of care in Egypt.

Concerning the causes of intra-hospital death, results revealed that main cause of death is circulatory in study group, while main cause of death is respiratory diseases in control group. This finding agree with Panula et al., (2011) \& Tarazona-Santabalbina et al., (2012) who found that death from circulatory disease is main cause in the studied sample. Also supported with McNicoll \& Fitzgibbons, (2009) who found main cause of death is respiratory diseases among elderly patients with hip fracture.

Concerning level of knowledge, results revealed that high statistical significant difference was found between study and control groups as regard patient's level of knowledge about hip fracture following the educational program. This finding agree with Lin et al., (2009) who found high statistical significant difference was found between study and control groups after implementing educational program.

\section{Conclusion}

Based on the result and research hypothesis of the present study, it concluded that; the most common type of hip fracture among studied sample is intertrochanteric fracture; the main cause of the hip fracture is fall down. This study revealed that, elderly patients had poor knowledge regarding hip fracture. After implementation of the educational program, the elderly patients' knowledge was improved.

\section{Recommendations}

1- Increase public awareness about hip fracture through expanded health education about the problem for elders at Sohag that can be achieved by health classes and home visits.

2- Periodic checkup of bone mineral density on physical examination for the elderly people is a requirement for the prevention hip fracture. 


\section{References}

1. Abd-Elmohsen S., (2013): Rehabilitation guidelines for patients undergoing arthroscopic knee surgery for meniscal trimming, Doctorate Thesis, Faculty of Nursing Assiut University.

2. Al-Faisal, W. (2007): Falls Prevention for Older Persons, Eastern Mediterranean Regional Review, $1-23$.

3. American Academy of Orthopedic Surgeons (AAOS), (2015): Hip Fractures. Available at www.orthoinfo.aaos.org/topic.com

4. Anderson, M., \& Wolfe, B., (2013): How Should Patients with Acute Hip Fractures Be Managed Preoperatively. Available at www.Hospitalist, November 2013

5. Beaupre, L., Cinats, J., Jones, A., Scharfenberger, A., Johnstonm D., Senthilselvanm A., \& Saundersm D., (2007): Does functional recovery in elderly hip fracture patients differ between patients admitted from long-term care and the community, Journal of Gerontology, Medical Sciences , The Gerontological Society of America, 62A (10): 1127-33.

6. Beaupre, L., Cinats, J., Senthilselvan, A., Scharfenberger, A., Johnston, W., \& Saunders, L., (2005): Does Standardized Rehabilitation and Discharge Planning Improve Functional Recovery in Elderly Patients With Hip Fracture, American Congress of Rehabilitation Medicine and the American Academy of Physical Medicine and Rehabilitation, 86 (1): 2231-9.

7. Beauprea, L., Binderb, E., Cameronc, I. Jonesa, A., Orwigd, D., Sherringtone, C., \& Magazinerf, J., (2013): Maximising functional recovery following hip fracture in frail seniors, Best Practice Research Clinical Rheumatology, 27 (6): 771-88.

8. Canadian Institute of Health Information (CIHI) (2015): Factors predicting return home from inpatient rehabilitation following hip fracture surgery, PP 1-22.

9. Casaletto, A., \& Gatt, R., (2004): Post-operative mortality related to waiting time for hip fracture surgery, Injury, 35 (2): 114-20.

10. Central Agency for Public Mobilization and Statistics (CAMPS), (2015): Vital Statistics, https://www.census.gov/prod/cen2010/briefs/c201 0br-09.pdf.

11. Colquhoun, A., Zuelzer, W., \& Butterworth, J., (2014): Improving the Management of Hip Fractures in the Elderly A Role for the Perioperative Surgical Home, the American Society of Anesthesiologists, Inc. Lippincott Williams \& Wilkins. Anesthesiology, Anesthesiology, 121 (6): 1144-6.
12. Dailiana, Z., Papakostidou, I., Varitimidis, S., Michalitsis, S., Veloni, A., \& Malizos, K., (2013): Surgical treatment of hip fractures: factors influencing mortality, Hippokratia 17 (3): 252-7.

13. Deka, R., Shetty, S., Shetty, M., Mohapatra, A., Poulose, P., \& Krishna, V., (2015): Functional Outcome of Dynamic Hip Screw in Elderly People: A Clinical Study, International Journal of Scientific Study, February 2 (11): 142-6.

14. El-Ghandour, S., (2014): Endoprosthetic replacement for failed internally fixed intertrochanteric hip fractures, Egyptian Orthopedic Journal, 1 (1): 49-68.

15. El-jedi, A., Mousa, A., \& Naim, F., (2015): Hip fracture and disabilities among elderly in Gaza Governorates, Palestine, Journal of Natural and Engineering Studies, 23 (1): 63-80.

16. Griffin, J., Anthony, T., Murphy, D., Brennan, K., \& Brennan, M., (2016): What is the impact of age on reoperation rates for femoral neck fractures treated with internal fixation and hemiarthroplasty, A comparison of hip fracture outcomes in the very elderly population, Journal of Orthopedic, 13(1): $33-9$.

17. Hacking, R., \& O'Connor, D., (2014): Anatomy and Physiology of Ageing, world anesthesia tutorial of the week. Available at www. AnesthesiaUK. Com/ world anesthesia.

18. Handoll, H., \& Parker, M., (2008): Conservative versus operative treatment for hip fractures in adults (Review), The Cochrane Collaboration Published by John Wiley, 1 (3): 8. Retrieved from http://www.thecochranelibrary.com

19. Hasegawa, Y., Suzuki, S., \& Wingstrand, H., (2007): Risk of mortality following hip fracture in Japan, Journal of Orthopedic Science, 12 (2):1137.

20. Hassan, S., (2015): A Study of Morbidity Pattern among Geriatric Population in Fayoum Governorate, Egypt, Journal of American Science 11 (4): P 90-5.

21. Herr, K., \& Titler, M., (2009): Acute pain assessment and pharmacological management practices for the older adult with a hip fracture, Journal of emergency nursing, 35 (4): 312-20.

22. Keswani, A., Lovy, A., Khalid, M., Blaufarb, I., Moucha, C., Forsh, D., \& Chen, D., (2016): The effect of aortic stenosis on elderly hip fracture outcomes, Injury, International journal of Care Injured 2016; 47 (1): 413-8.

23. Kiel, D., Magaziner, J., Zimmerman, S., Ball, L., Barton, B., Brown, K., Stone J., Stone D., \& Birge S., (2007): Efficacy of a Hip Protector to 
Prevent Hip Fracture in Nursing Home Residents, 298 (4): 413-22.

24. Kim S., Moon Y., Lim S., Yoon B., Min Y., Lee D., \& Park Y., (2012): Prediction of survival, second fracture, and functional recovery following the first hip fracture surgery in elderly patients, Bone, 50 (1):1343-50.

25. Kondo, A., Sada, K., Yamaguchi, C., \& Fujimoto, E., (2012): Nursing Care after Hip Fracture Surgery Predicts Patient Ambulatory Ability at 3 Months after Surgery, Professional Issues and Trends in Nursing in Japan, PP2167-8.

26. Larsson, G., Strömberg, U., Rogmark, C., \& Nilsdotter, A., (2016): Prehospital fast track care for patients with hip fracture: impact on time to surgery, hospital stay, postoperative complications and mortality A randomized, controlled trial, Injury, International Journal of Care Injured, 1(1): 1-22.

27. Liao, C., Lin, C., Shih, C., Yeh, C., Chang, Y., Lee, Y., \& Chen, T., (2014): Increased Risk of Fracture and Post fracture Adverse Events in Patients with Diabetes: Two Nationwide Population-Based Retrospective Cohort Studies, Diabetes Care, 37(1): 2246-52.

28. Lin, C., Wang, H., Chen, S., Liao, P., Kao, F., \& Wu, F., (2009): To evaluate the effectiveness of discharge planning programme for hip fracture patients, Journal of clinical Nursing, 18 (11): 1632-9.

29. Lin, P., \& Chang, S., (2004): Functional recovery among elderly people one year after hip fracture surgery, Journal of Nursing Research, 12 (1): 72-80.

30. Liu, H. Yang, C., Cheng, H., Wu, C., Chene, C., \& Shyu, Y., (2015): Family caregivers' mental health is associated with postoperative recovery of elderly patients with hip fracture, a sample in Taiwan, Journal of Psychosomatic Research, 78 (1): 452-8.

31. Maggi, S., Siviero, P., Wetle, T., Besdine, R., Saugo, M., \& Crepaldi, G., (2010): A multicenter survey on profile of care for hip fracture: predictors of mortality and disability, International Osteoporosis Foundation and National Osteoporosis Foundation, 21(1): 223-31.

32. Maher, A., Meehan, A., Hertz, K., MacDonald, V., Sullivan, M., Specht, K.and Taylor, A., (2012): Acute nursing care of the older adult with fragility hip fracture: An international perspective (Part 2), International Journal of Orthopaedic and Trauma Nursing; 1: 1-15. Retrieved from www.elsevier.com/locate/ijotn

33. Makridis, K., Karachalios, T., Kontogeorgakos, V., Badras, L., \& Malizos, K., (2014): The effect of osteoporotic treatment on the functional outcome, re-fracture rate, quality of life and mortality in patients with hip fractures: A prospective functional and clinical outcome study on 520 patients, Injury, International journal of Care Injured, 1 (1): 1-6.

34. Matre, K., (2013): Treatment of trochanteric and subtrochanteric hip fractures Sliding hip screw or intramedullary nail, Dissertation for the degree of philosophiae doctor $(\mathrm{PhD})$ at the University of Bergen, Introduction and background, PP12-9.

35. Matsui, Y., Harada, A., Takemura, M., Terabe, Y., \& Hida, T., (2014): Falls in hip fracture patients: relation between fall situations and ADL capability before injury, Journal of Osteoporosis \& Physical Activity, 2 (1): 108.

36. McCallum J. (2011): Ageing research directions for Australia, Austral Journal Ageing 2011, 3: 13.

37. McGilton, K. Davis, A. Naglie, G. Mahomed, N. Flannery, J. Jaglal, S. Cott, C. and Stewart, S. (2013): Evaluation of patient-centered rehabilitation model targeting older persons with a hip fracture including those with cognitive impairment, BMC Geriatrics;13 (136): 1-8.

38. McGilton, K., Mahomed, N., Davis, A., Flannery, J., \& Calabrese, S., (2015): Outcomes for Older Adults in an Inpatient Rehabilitation Facility Following Hip Fracture Surgery, PP 30-3.

39. McNicoll, L., \& Fitzgibbons, P., (2009): Optimal hip fracture management in high-risk frail older adults, Geriatrics for the Division of geriatrics practicing physician, 92 (7): 250-1.

40. Nagai, T., \& Okawa, A., (2016): Risk factors affecting postoperative walking ability following hip fracture surgery in the elderly, Journal of Orthopedic and Muscular System, 5 (2): 209-5.

41. Orwig, D., Hochberg, M., Yu-Yahiro, J., Resnick, B., Hawkes, W., Shardell, M., Hebel, J., Colvin, P., Miller, R., Golden, J., Zimmerman, S., \& Magaziner, J., (2011): Delivery and Outcomes of a Yearlong Home Exercise Program After Hip Fracture, Arch Internal Medicine, 171 (4): 323-31.

42. Panula, J., (2010): Surgically treated hip fracture in older people with special emphasis on mortality analysis, mortality and causes of death of hip fracture patients, university of Turku, PP 28-33.

43. Paruk, F., \& Cassim, B., (2012): Osteoporotic Hip Fractures Risk factors and Outcomes at one year eThekwini region: South Africa, 1st Africa Region Congress of Gerontology and Geriatrics Cape Town, October, Department of Geriatrics University of KwaZulu-Natal.

44. Paula, F., Cunha, G., Leite, I., Pinheiro, R., \& Valente, J., (2015): Elderly readmission and 
death after discharge from treatment of hip fracture occurred in public hospitals from 2008 to 2010, epidemiology Journal 18 (2): 439-53.

45. Perneczky, R., Wagenpfeil, S., Komosa, K., Grimmer, T., Diehl, J., \& Kurz, A., (2006): Mapping scores onto stages, mini-mental and clinical dementia rating, American Journal Geriatric Psychiatric, 14 (1): 139-44.

46. Shakhatreh, H., (2001): Analysis of fractures of the proximal femur in the Jordanian population. Israel Medical Association Journal, 3 (1): 28-31.

47. Shyu, Y., Liang, J., Tseng, M., Li, H., Wu, C., Cheng, H., Yang, C., \& Chou, S., (2012): Comprehensive Care Improves Health Outcomes among Elderly Taiwanese Patients With Hip Fracture, Journals of Gerontology: Medical Sciences 68 (2): 188-97.

48. Shyu, Y., Liang, J., Wu, C., Su, Y., Cheng, S., Chou, W., Chen, C., \& Yang, T., (2008): Interdisciplinary intervention for hip fracture in older Taiwanese: benefits last for 1 year, Journals of Gerontology, Medical Sciences,63 (1): 92-7.

49. Soveid, M. Serati, R. and Masoompoor, M. (2005): Incidence of hip fracture in Shiraz, Iran, Osteoporosis; 16 (11): 1412.

50. Stenvall, M., Berggren, M., Lundstro, M., Gustafson, Y., \& Olofsson, B., (2012): A multidisciplinary intervention program improved the outcome after hip fracture for people with dementia Subgroup analyses of a randomized controlled trial, Archives of Gerontology and Geriatrics, 54 (2012): 284-9.

51. Tarazona- Santabalbina, F., Belenguer-Varea, A., Rovira-Daudi, E. Salcedo-Mahiques, E., Cuesta-Peredo,' D., Salvador-Pe'rez, M., \& Avellana-Zaragoza, J., (2012): Early interdisciplinary hospital intervention for elderly patients with hip fractures functional outcome and mortality, Clinics 67 (6): 547-55.

52. Vergara, I., Vrotsou, K., Orive, M., Gonzalez, N., Garcia, S., \& Quintana, J., (2014): Factors related to functional prognosis in elderly patients after accidental hip fractures, BMC Geriatrics 14 (124): 1-7.

53. Vochteloo, A., Moerman, S., Tuinebreijer, W., Maier, A., Vries, M., Bloem, R. Nelissen, R., \& Pilot. P., (2013): More than half of hip fracture patients do not regain mobility in the first postoperative year, Geriatrics Gerontology International, 13 (1): 334-41.

54. Yoon, K., Park, C., Jang, S., Lee, K., \& Ha, C., (2011): Incidence and mortality following hip fracture in Korea, Journal Korean Medical Science, 26 (1): 1087-92.

55. Zapatero, A., Barba, R., Canora, J., Losa, J., Plaza, S., Roman, J., \& Marco, J., (2013): Hip fracture in hospitalized medical patients, Journal of BMC Musculoskeletal Disorders, 1 (1): 1-6.

56. Zheng, Y., Ruan, G., Fu, J., Su, Z., \& Lu, J., (2016): Postoperative plasma 8-iso-Prostaglandin F2 $\alpha$ levels are associated with delirium and cognitive dysfunction in elderly patients after hip fracture surgery, 4-10. 